\title{
Sequences of Sequences: Spatial Organization of Coded Matter through Layer-by-layer Assembly of Digital Polymers.
}

\author{
Roza Szweda, Michel Tschopp, Olivier Felix, Gero Decher* and Jean-François Lutz* \\ Université de Strasbourg, CNRS, Institut Charles Sadron UPR22, 23 rue du Loess, 67034 Strasbourg Cedex 2, \\ France
}

Keywords: data storage, digital materials, information-containing macromolecules, layer-bylayer, sequence-controlled polymers

\begin{abstract}
A library of 16 digitally encoded polyanions was used in a layer-by-layer (LbL) polyelectrolyte assembly to nanofabricate thin films containing digitally coded strata. The polyanions were digital polyphosphodiesters (d-PPDE) prepared via an automated phosphoramidite process. Each component of the library contained 10 bytes of ASCII-encoded text (i.e. 80 coded monomers); thus the entire library allows the writing of a full sentence, which can be stored in a multilayer film as a sequence of sequences. To prepare fully segregated digital domains, non-coded layers composed of poly(allylamine hydrochloride) (PAH)/poly(sodium 4styrenesulfonate) (PSS) were included between the d-PPDE coded layers as an intermediate barrier. Detailed analysis of the film homogeneity indicated formation of $70 \mathrm{~nm}$-thick films in which digital layers are kept apart from another by non-coded interlayers. As a result, the sequence-coded polymer library could be piled-up in a defined sequence of layers.
\end{abstract}

It was shown in recent years that the monomer sequence of copolymers can be used to store information.[1] Polymers having such a property are called information-containing macromolecules, [1b] or more specifically digital polymers when they store digital information. In DNA, for example, the four nucleotides of the genetic alphabet can be used to write binary messages, as shown in several recent reports.[2] Alternatively, molecular information can be encrypted in the comonomer sequence of non-biological polymers.[3] The information stored inside the polymer chains can be recovered using a sequencing technique, such as highthroughput Illumina sequencing, nanopore sequencing, or tandem mass spectrometry.[4] Some strategies for erasing or rewriting coded monomer sequences have also been reported.[3c, 5] Thus, it is now clearly demonstrated that information sequences can be stored, manipulated, and retrieved at the single-polymer-chain level. Yet, beyond these interesting discoveries, there is currently very little research about the spatial organization of man-made coded matter. In the bulk, digital polymers contain a very large amount of uniform chains having all the same sequence-encoded message. However, potential applications of these polymers, for example, data storage, will probably require the development of organized polymer libraries in which a piece of information can be stored in a defined region of space. For nucleic acids memories, commercial DNA microchips are usually purchased and used as such,[2a,c] which is easy but probably not an optimal solution. Indeed, modern materials science offers many other opportunities to organize soft matter at the nanoscale. For instance, the 2D or 3D organization of digital polymer libraries could be attained via surface patterning, directed self-assembly, co-crystallization, layer-by-layer (LbL) deposition, or by a combination of some of these approaches. 
In this context, we report herein the LbL preparation of multilayered thin films containing segregated digital domains. During the last two decades, the LbL process has been shown to be an extremely simple and versatile platform for preparing structured nanomaterials. [6] In the original version of it, oppositely charged polyelectrolytes (that is, polyanions and polycations) are repeatedly deposited on a charged substrate with a nanometer precision to form a thin film containing alternating polyanion/polycation layers. However, the LbL concept has been afterwards extended to many other types of associating polymers and materials, [7] thus enabling preparation of a broad variety of nanostructured materials including colloids, organic/inorganic composites and bio-hybrid structures.[8] Furthermore, the deposition process can be facilitated through the use of automated dipping, spin- or spray-assisted assembly.[9] Yet, although it was already realized in the early days of LbL-assembly that a given sequence of different dipping solutions leads to a unique layer sequence,[6a,10] many people in the field continued to prepare LbL-films containing only two components in strictly alternating fashion. In the present work, a library of digitally encoded polyelectrolytes was used to our knowledge for the first time for the fabrication of LbL films in which each digital layer contains a different piece of coded information (Figure 1). In other words, the information stored in these materials is determined by the monomer sequence of a given digital polymer in a given layer but also by the sequential arrangement of the coded layers in the entire film. The formation of such a "sequence of sequences" was studied herein using ellipsometry, atomic force microscopy (AFM), and X-ray photoelectron spectroscopy (XPS).
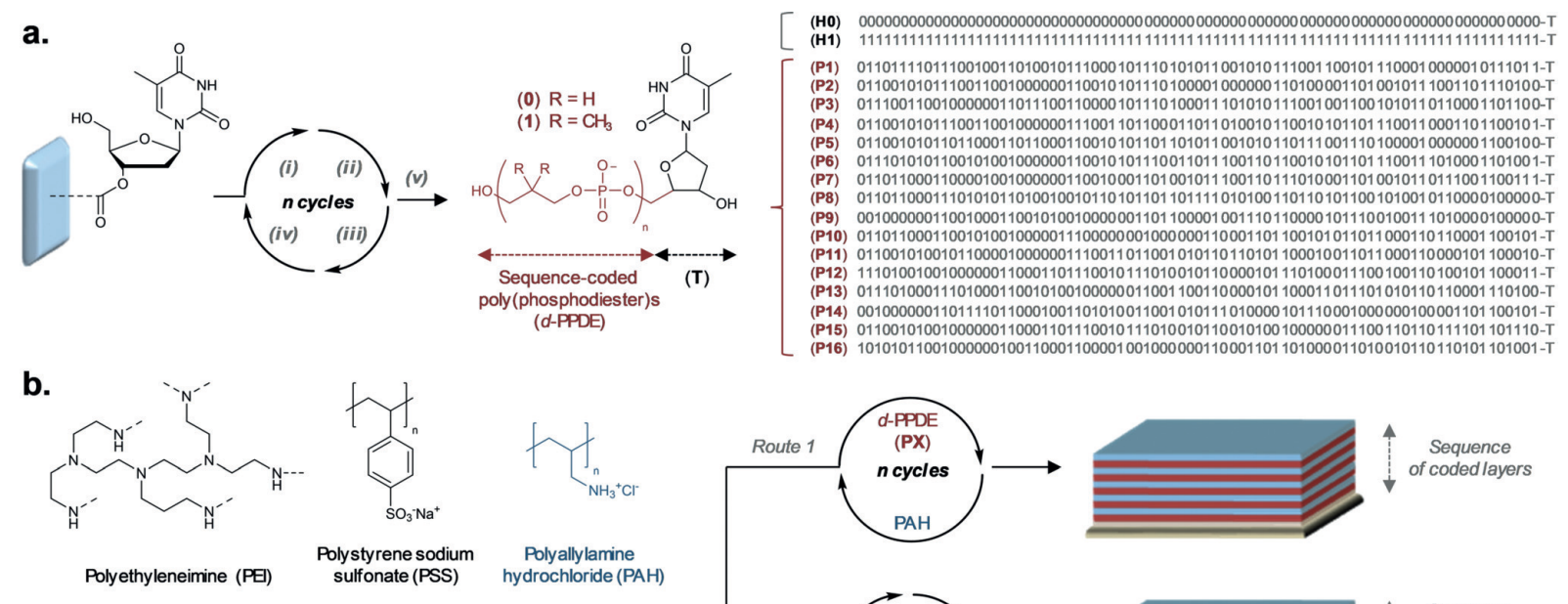

c.
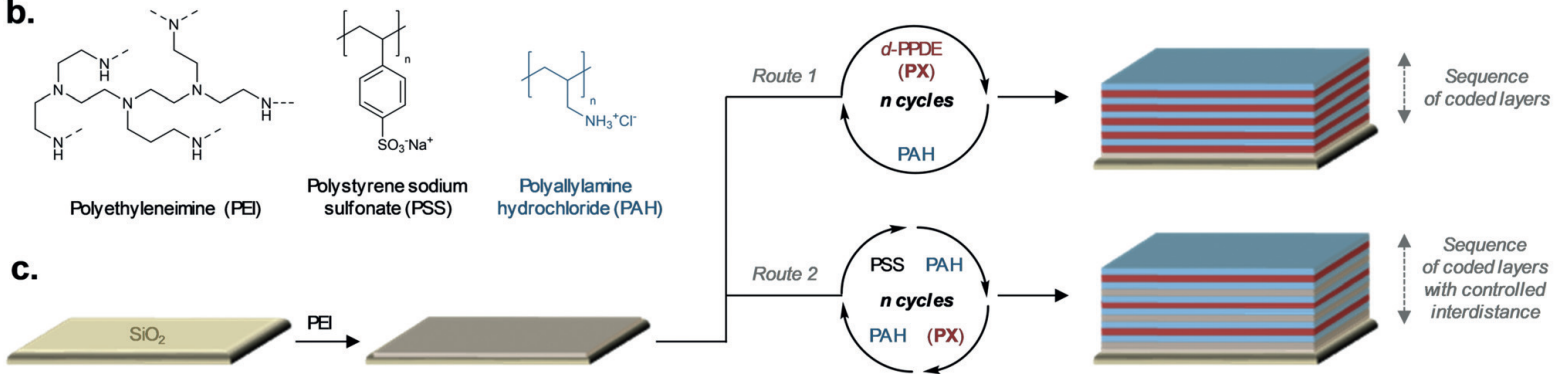

Figure 1. General strategy studied in this work for the preparation of digital multilayers. a) Synthesis of a library of sequence-coded polyanions by solid-phase phosphoramidite chemistry. Experimental conditions: i) phosphoramidite coupling: $R T$, acetonitrile, tetrazole; ii) oxidation: $R T, 12$, pyridine, THF/H2O; iii) capping: RT, acetic anhydride, $\mathrm{N}$-methylimidazole; iv) DMT deprotection: $\mathrm{CCl} 3-\mathrm{COOH} ; \mathrm{CH} 2 \mathrm{Cl} 2$; v) cleavage: $\mathrm{MeNH}$, $\mathrm{NH} 4 \mathrm{OH}$, $H 2 O, R T$. The blue square on the right side represents a porous glass solid support. The decabyte monomer sequences of the homopolymers (HO and $\mathbf{H 1}$ ) and copolymers (P1-P16) synthesized in this work are listed on the right. b) Molecular structures of non-coded polyanions and polycations that were used in the layer-by-layer process. c) Preparation of digital multilayers. Route 1: alternating deposition of coded d-PPDE (red) and noncoded PAH (blue). Route 2: four-step PSS/PAH/d-PPDE/PAH deposition cycle.

Digital polyphosphodiesters (d-PPDE) were selected in the present work as coded polyanions. As recently described,[3b] these polymers are obtained via a solid-phase phosphoramidite chemistry approach in which two different phosphoramidite monomers are used as a binary 
alphabet (Figure 1a). Following previously established conventions, monomers containing a propyl and a 2,2-dimethylpropyl spacer are defined as 0- and 1-bit, respectively.[11] Thus, the synthesis and sequencing of long digital polyphosphodiesters has been previously demonstrated.[12] Herein, a library of 16 different d-PPDEs was synthesized. This library encodes a citation from the French chemist Marcellin Berthelot: "La chimie crée son objet. Cette faculté créatrice, semblable à celle de l'art lui-même, la distingue essentiellement des sciences naturelles et historiques." (Supporting Information, Figure S1).[13] This sentence contains 160 characters, including spaces and quotation marks. Thus, using extended ASCIIencoding, it was decomposed into 16 different polymers P1-P16 containing each 10 bytes of information (Supporting Information, Table S1; Figure 1a).[14] Each polymer contained therefore 81 monomers, that is, 80 binary monomers (one byte of information corresponds to 8 monomers) and one thymidine nucleoside ( $T$ ) coming from the porous glass solid support. All these polymers were synthesized on an automated synthesizer and their structural uniformity was assessed by HPLC (Supporting Information, Figure S2a) and NMR (Supporting Information, Figure S3). Furthermore, two homopolymers $\mathbf{H O}$ and $\mathbf{H 1}$ with the same chainlength as the copolymers of the library but containing only 0 or 1 units were synthesized and characterized (Supporting Information, Figures S2b and S3).

The LbL assembly process was first studied with the model homopolyanions $\mathbf{H O}$ and $\mathbf{H 1}$, using poly(allylamine hydrochloride) (PAH) as a counter-polycation (Figure 1b). These first experiments were performed to assess the influence of the nature of the coded monomer on film formation. Another important aspect of the present work was to avoid non-linear LbL growth, which has already been observed with some polyphosphates.[15] Indeed, superlinear growth leads to an enhanced diffusion process and therefore to the migration of polyelectrolyte between layers.[16] Such a behavior would not be ideal for the present study in which coded digital layers should be completely segregated from another. Thus, $\mathbf{H} \mathbf{0}$ and $\mathbf{H} \mathbf{1}$ were first studied to identify experimental conditions, in which the films grow homogenously and linearly. The polyelectrolyte films were constructed on a modified silicon wafers by a manual dipping method and their growth was followed by ellipsometry (Supporting Information, Figure S4). First, a simple alternating process (Route 1 in Figure 1c) was investigated in which a PAH cationic layer was added in between each d-PPDE layer. These experiments showed that the molecular structure of the homopolymers has an influence on $\mathrm{LbL}$ growth and on the properties of the resulting films. When using $\mathbf{H O}$ as a polyanion (Supporting Information, Figure S4a), LbL growth followed a linear regime for all investigated concentrations. For instance, after depositing $10 \mathrm{PAH} / \mathrm{HO}$ layers at a polyelectrolyte concentration of $0.04 \%$, thin films with an overall thickness of $5 \mathrm{~nm}$ and a root-mean-square (RMS) roughness of $1.1 \mathrm{~nm}$ were obtained. In contrast, a superlinear growth was observed for films prepared with $\mathbf{H 1}$ (Supporting Information, Figure S4b).[17] After 10 PAH/H1 depositions at a concentration of $0.04 \%$, ellipsometry evidenced formation of films with an overall thickness of $38 \mathrm{~nm}$, thus suggesting extended and well-hydrated structures, in which chain migration between layers might occur.[16,17] Film inhomogeneity was confirmed by AFM measurements, which indicated an RMS roughness of about $5.7 \mathrm{~nm}$. These results underline that, despite the small structural differences between monomers 0 and 1 (that is, two protons instead of two $\mathrm{CH} 3$ groups), the composition extremes found in $\mathbf{H O}$ and $\mathbf{H} \mathbf{1}$ lead to different LbL behaviors. Furthermore, when $\mathbf{H O}$ and $\mathbf{H 1}$ were both mixed in the same film (Supporting Information, Figure S5), non-linear growth was still observed, thus confirming the dominating role of $\mathbf{H} \mathbf{1}$. 
To minimize these discrepancies, a second deposition strategy (Route 2 in Figure 1c) was investigated with $\mathbf{H O}$ and $\mathbf{H 1}$. In this approach, a complementary polyanion, poly(sodium 4styrenesulfonate) (PSS) was also used (Figure 1b), thus enabling to include non-coded PSS/PAH interlayers between coded d-PPDE layers. PSS was chosen as a copolyanion because $\mathrm{PSS} / \mathrm{PAH}$ is a widely investigated and well understood polyelectrolyte pair in the LbL field.[6a, 9c] XPS measurements that were performed on model surfaces, on which PSS, PAH, and a dPPDE homopolymer were successively deposited are shown in the Supporting Information, Figure S6. These results show that $\mathbf{H O}$ and $\mathbf{H 1}$ can be deposited on PSS/PAH interlayers. Furthermore, when inserting one PSS interlayer between two d-PPDE layers (that is, using a four-step deposition cycle as shown on Figure 1b), a linear growth was observed with both homopolymers $\mathbf{H O}$ and $\mathbf{H 1}$ (Supporting Information, Figure S7). For instance, after performing 10 cycles, both homopolymers led to comparable film having a thickness of $45 \mathrm{~nm}$ and a RMS roughness of $2 \mathrm{~nm}$. Hence, Route 2 prevents non-linear LbL growth and was therefore selected for the preparation of digital multilayers.

a.
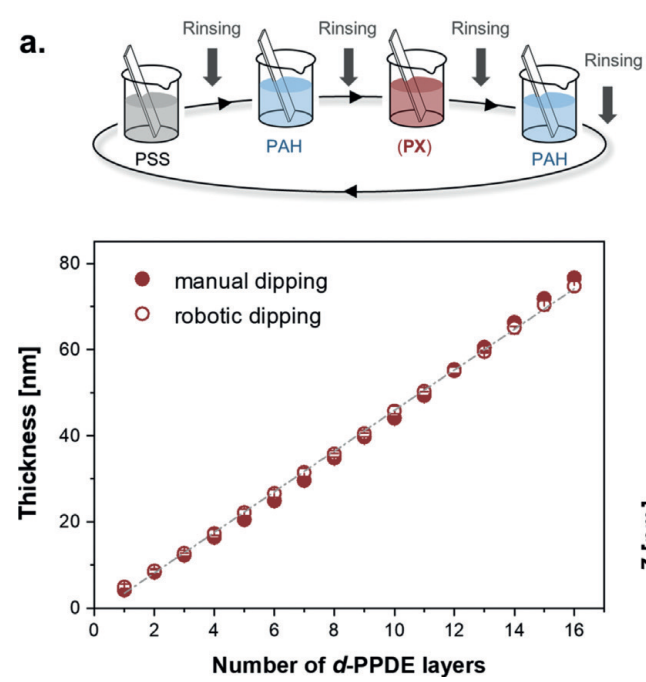

b.

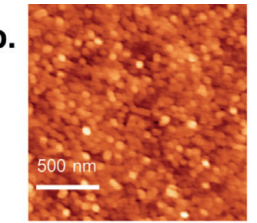

c.
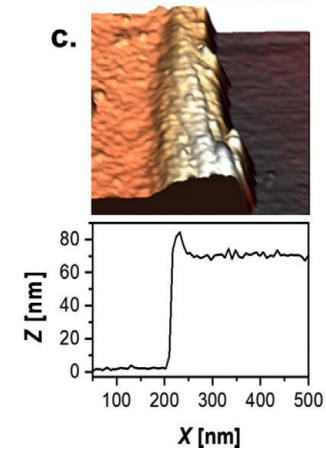

d.
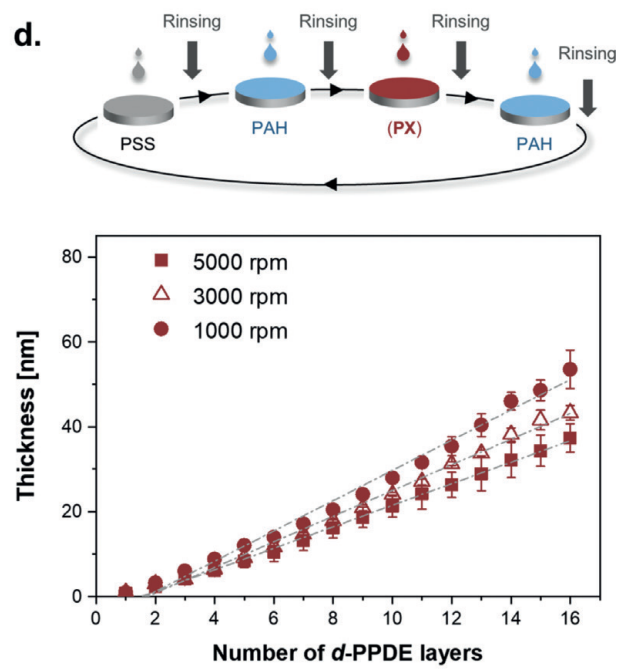

Figure 2. Characterization of digital multilayers prepared by dipping and spin-assisted LbL approaches. a) top: representation of the dipping protocol (route 2, Figure 1c); bottom: ellipsometry monitoring of film thickness for multilayers prepared by either manual $(\bullet)$ or robotic (O) LbL dipping. The studied films contained 16 coded d-PPDE layers (Supporting Information, Table S1); that is, 63 layers in total. Ellipsometry measurements were made after each d-PPDE deposition. The error bars represent standard deviation values of three independent measurements performed at different surface points. b) AFM characterization of the surface morphology of the final film prepared by robotic dipping. The size of AFM images is $1.5 \times 1.5 \mathrm{~mm}$ and Z scale is $15 \mathrm{~nm}$. c) AFM cross-section measured for the final film obtained by robotic dipping. d) top: representation of the spin-assisted assembly protocol (route 2, Figure 1c); bottom: ellipsometry monitoring of film thickness for films prepared spin-assisted $L b L$ assembly at different rotational speeds. The films contained 16 d-PPDE layers and the ellipsometry measurements were made after each d-PPDE deposition. Errors bars were estimated as in (a).

The digital multilayers were first prepared by a conventional dipping LbL process as shown on Figure 2a.[6a] Following Route 2, a four-step PSS/PAH/d-PPDE/PAH deposition cycle was used. Consequently, 63 consecutive layers were deposited to include the 16 coded polyanions P1P16 of the d-PPDE library (Supporting Information, Table S1). The sequence of coded layers was arbitrarily arranged from the top to the bottom of the film. In other words, d-PPDE deposition started with P16 and finished with P1 (that is, following the reverse order of Table S1). The LbL dipping approach was first performed manually to carefully study film formation. Yet, an important aim of the present work was to demonstrate that the preparation of digital films can also be automated. Thus robotic LbL dipping was also studied and compared to the 
manual approach. In both cases, similar polyelectrolyte deposition protocols were used. Figure 2a shows the evolution of film thickness determined by ellipsometry as a function of the number of deposited d-PPDE layers for both manual and robotic dipping approaches. As previously observed with homopolymers $\mathbf{H O}$ and $\mathbf{H 1}$, a linear growth was observed in all cases. It shall be specified that the molar fraction F0 of comonomer 0 in copolymers P1-P16, is in a range $0.46-0.63$ (Supporting Information, Table S1), which is intermediate as compared to the previously studied extremes $\mathbf{H O}$ and $\mathbf{H 1}$. It is therefore expected that copolymers follow a similar LbL trend as homopolymers, even though a defined sequence of layers was created using P1-P16. Furthermore, the film thicknesses measured by ellipsometry at all stages of the LbL growth were similar for manual and robotic approaches, thus confirming that automated deposition can be safely employed to prepare digital films. Altogether, the ellipsometry results of Figure 2a suggest that there is limited polyelectrolyte diffusion between layers and that $d$ PPDE are physically segregated from another.

This assumption was further supported by the AFM analysis of film morphology (Figure $2 b, c$; Supporting Information, Figure S8a). The manual and robotic LbL protocols led to final films (that is, films containing 63 polyelectrolyte layers including $16 \mathrm{~d}$-PPDE coded layers) of similar morphology with comparable RMS roughness values of 2 and $1.8 \mathrm{~nm}$, respectively. The slightly lower RMS value obtained for the robotic protocol may be explained by its more precise sample treatment, eliminating unnecessary manipulations and reducing the probability of scratches or other film damage. For both manual and robotic approaches, the film morphologies appeared homogenous for the whole area studied by AFM, which is in agreement with the small thickness error values estimated by ellipsometry. Furthermore, AFM "scratching" (Figure 2c; Supporting Information, Figure S8a) was used to measure final film thickness. For instance, for the digital films prepared via the robotic approach (Figure 2c), an overall thickness of $70 \mathrm{~nm}$ was measured, which is in good accordance with the ellipsometry estimations $(75 \mathrm{~nm})$. Hence, the AFM data suggest a controlled inter-distance of about $3 \mathrm{~nm}$ between each d-PPDE layer. If needed, this distance can be, of course, enlarged by increasing the number of PSS/PAH non-coded interlayers.

The fabrication of digital multilayers was also investigated using spin-assisted deposition, which is an efficient method for facilitating deposition and reducing preparation times.[9b] The films were formed by sequentially dropping polyelectrolyte and rinsing solutions on a modified silicon wafer placed on a spinning unit (Figure $2 \mathrm{~d}$, top). Route 2 (Figure $1 \mathrm{c}$ ) was used to form the multilayers and a short spinning time of $30 \mathrm{~s}$ was used for each polyelectrolyte deposition. The LbL deposition was studied at different rotational speeds $(1000,3000$, and $5000 \mathrm{rpm}$ ). As evidenced by ellipsometry monitoring (Figure $2 \mathrm{~d}$, bottom), all resulting films showed linear growth. However, the spinning speed influenced the thickness, homogeneity and roughness of the resulting materials. First of all, overall film thickness increased with decreasing rotational speed, which is due to a longer contact time between polyelectrolyte solution and substrate at slow rotation rates. Regarding film homogeneity, a fast spinning rate (5000 rpm) led to a marked decrease of film thickness at the central point of the sample due to the limited contact time of the polyelectrolyte solution at the midpoint of the substrate. On the other extreme, the use of a slow spinning rate $(1000 \mathrm{rpm})$ results in a poor spreading of polyelectrolyte solutions on the substrate and therefore in the formation of nonhomogeneous films (see errors bars in Figure 2d). Optimal results were obtained with an intermediate spinning speed of $3000 \mathrm{rpm}$, at which rotation was fast enough to homogenously distribute the polyelectrolyte solutions while maintaining comparable solution-surface contact time over the entire surface area. At this rate, homogenous films with a RMS 
roughness of only $1.4 \mathrm{~nm}$ were obtained (Supporting Information, Figure S8). Thus, spinassisted LbL assembly is a suitable approach for the preparation of digital multilayers. Furthermore, this method is overall much faster than dipping approaches. Indeed, the fabrication of thin films containing 16 coded d-PPDE layers required about $1 \mathrm{~h}$ by spin-assisted deposition, whereas it takes about one day by dipping. Yet, the formed films are thinner (40 $\mathrm{nm}$ at $3000 \mathrm{rpm}$ ) than those prepared by the dipping approach and therefore the interdistance between two coded layers is only of about $2 \mathrm{~nm}$. Of course, as pointed out above, this distance could likely be increased by the use of additional PSS/PAH layers.

In summary, thin films containing segregated layers of digitally encoded polymers were prepared by LbL and characterized. An ASCII-encoded sentence of 160 bytes was stored in these films using a library of 16 different digital polyanions. The storage multilayers were prepared using various LbL methods such as manual dipping, robotic dipping, and spinassisted deposition. All three approaches allowed synthesis of thin films with controlled thickness, roughness, and morphology. However, it was found in this work that the use of noncoded PSS/PAH intermediate layers is mandatory to isolate efficiently coded digital layers from another. Overall, this work opens up very interesting new avenues in fundamental and applied polymer science. From a fundamental point of view, the present results highlight for the first time that abiotic coded matter can be organized in precise nanoscale morphologies. Moreover, the formation of a "sequence of sequences" increases coding possibilities in synthetic materials. For instance, out of the $16^{16}$ possible multilayers that could have been created using a set of 16 digital polymers in 16 coded layers, only the sequence reported here gives access to Berthelot's quote (assuming film directionality). Regarding potential applications, the strategies described here could be relevant for the development of molecular archives and other data storage applications. For instance, the combination of 2D patterning ( $x$-y organization) with LbL growth (z-organization) could lead to storage materials with unprecedented storage densities. Yet, digital polymers have been barely studied in materials science and nanotechnology so far and many scientific aspects still have to be addressed. For instance, the development of sequencing methods allowing spatial decoding of coded libraries is an exciting new challenge in polymer analytics. In the case of digital LbL films, decoding could be achieved using depth profiling mass spectrometry methods such as time-of-flight secondary ion mass spectrometry (TOF-SIMS) or desorption electrospray ionization (DESI).[18]

\section{ASSOCIATED CONTENT}

\section{Supporting Information}

Full experimental part: detailed experimental procedures. Supplementary Figures S1-S7.

\section{AUTHOR INFORMATION}

\section{Corresponding Author}

Jean-François Lutz, E-mail: jflutz@unistra.fr

Gero Decher, E-mail: decher@unistra.fr

\section{ACKNOWLEDGMENT}


J.F.L. thanks the H2020 program of the European Union (project Euro-Sequences, H2020MSCA-ITN-2014, grant agreement no. 642083) and the CNRS for financial support. The authors thank Pierre Schaaf (Inserm 1121, Strasbourg) for the access to the AFM.

\section{REFERENCES}

[1] a) J.-F. Lutz, M. Ouchi, D. R. Liu, M. Sawamoto, Science 2013, 341, 1238149; b) H. M. Colquhoun, J.-F. Lutz, Nat. Chem. 2014, 6, 455 -456; c) J.-F. Lutz, Macromolecules 2015, 48, $4759-4767$.

[2] a) V. Zhirnov, R. M. Zadegan, G. S. Sandhu, G. M. Church, W. L. Hughes, Nat. Mater. 2016, 15, 366-370; b) C. Mayer, G. R. Mclnroy, P. Murat, P. Van Delft, S. Balasubramanian, Angew. Chem. Int. Ed. 2016, 55, 11144 - 11148; Angew. Chem. 2016, 128, 11310 - 11314; c) Y. Erlich, D. Zielinski, Science 2017, 355, 950; d) S. L. Shipman, J. Nivala, J. D. Macklis, G. M. Church, Nature 2017, 547, $345-349$.

[3] a) T. T. Trinh, L. Oswald, D. Chan-Seng, J.-F. Lutz, Macromol. Rapid Commun. 2014, 35, 141 - 145; b) A. Al Ouahabi, L. Charles, J.-F. Lutz, J. Am. Chem. Soc. 2015, 137, 5629 - 5635; c) R. K. Roy, A. Meszynska, C. Laure, L. Charles, C. Verchin, J.- F. Lutz, Nat. Commun. 2015, 6, 7237; d) G. Cavallo, A. Al Ouahabi, L. Oswald, L. Charles, J.-F. Lutz, J. Am. Chem. Soc. 2016, 138, 9417 - 9420; e) U. S. Gunay, B. E. Petit, D. Karamessini, A. Al Ouahabi, J.-A. Amalian, C. Chendo, M. Bouquey, D. Gigmes, L. Charles, J.-F. Lutz, Chem 2016, 1, $114-126$; f) N. Zydziak, W. Konrad, F. Feist, S. Afonin, S. Weidner, C. Barner-Kowollik, Nat. Commun. 2016, 7, 13672; g) A. C. Boukis, K. Reiter, M. Frölich, D. Hofheinz, M. A. R. Meier, Nat. Commun. 2018, 9, 1439.

[4] a) H. Mutlu, J.-F. Lutz, Angew. Chem. Int. Ed. 2014, 53, 13010-13019; Angew. Chem. 2014, 126, 13224-13233; b) J. Shendure, H. Ji, Nat. Biotechnol. 2008, 26, 1135 - 1145; c) L. Charles, C. Laure, J.-F. Lutz, R. K. Roy, Macromolecules 2015, 48, 4319 - 4328; d) J.-A. Amalian, T. T. Trinh, J.-F. Lutz, L. Charles, Anal. Chem. 2016, 88, 3715 - 3722.

[5] J. Bonnet, P. Subsoontorn, D. Endy, Proc. Natl. Acad. Sci. USA 2012, 109, 8884.

[6] a) G. Decher, Science 1997, 277, 1232-1237; b) G. Decher, J. B. Schlenoff, Multilayer Thin Films: Sequential Assembly of Nanocomposite Materials, 2nd ed., Wiley VCH, Weinheim, 2012. [7] E. Seyrek, G. Decher, in Polymer Science: A Comprehensive Reference, Vol. 7 (Eds.: K. Matyjaszewski, M. Möller), Elsevier, Amsterdam, 2012, pp. 159 - 185.

[8] a) F. Caruso, Adv. Mater. 2001, 13, 11- 22; b) P. T. Hammond, Adv. Mater. 2004, 16, 1271 -1293; c) J. J. Richardson, J. Cui, M. Björnmalm, J. A. Braunger, H. Ejima, F. Caruso, Chem. Rev. 2016, 116, 14828-14867; d) F.-X. Xiao, M. Pagliaro, Y.-J. Xu, B. Liu, Chem. Soc. Rev. 2016, 45, $3088-3121$.

[9] a) J. B. Schlenoff, S. T. Dubas, T. Farhat, Langmuir 2000, 16, 9968 - 9969; b) J. Cho, K. Char, J. D. Hong, K. B. Lee, Adv. Mater. 2001, 13, 1076 - 1078; c) A. Izquierdo, S. S. Ono, J. C. Voegel, P. Schaaf, G. Decher, Langmuir 2005, 21, $7558-7567$.

[10] a) A. A. Mamedov, N. A. Kotov, Langmuir 2000, 16, 5530-5533; b) K. Ariga, J. P. Hill, Q. Ji, Phys. Chem. Chem. Phys. 2007, 9, $2319-2340$.

[11] A. Al Ouahabi, M. Kotera, L. Charles, J.-F. Lutz, ACS Macro Lett. 2015, 4, 1077 - 1080.

[12] a) N. F. König, A. Al Ouahabi, S. Poyer, L. Charles, J.-F. Lutz, Angew. Chem. Int. Ed. 2017, 56, 7297 - 7301; Angew. Chem. 2017, 129, 7403 - 7407; b) A. Al Ouahabi, J.-A. Amalian, L. Charles, J.-F. Lutz, Nat. Commun. 2017, 8, 967.

[13] M. Berthelot, La Synthèse Chimique, Baillère, Paris, 1876.

[14] It is important to mention that the coded polyanions selected in this work are not optimal structures for MS/MS decoding. As reported in Reference [12b], poly(phosphodiester)s 
containing programmed fragmentation sites are mandatory for efficient sequencing. However, the purpose of this initial work being LbL film construction, we chose to work with robust polyelectrolytes that do not contain easily breakable bonds.

[15] N. Cini, T. Tulun, G. Decher, V. Ball, J. Am. Chem. Soc. 2010, 132, $8264-8265$.

[16] C. Picart, P. Lavalle, P. Hubert, F. J. G. Cuisinier, G. Decher, P. Schaaf, J. C. Voegel, Langmuir 2001, 17, $7414-7424$.

[17] For detailed information about linear and superlinear growth in LbL assembly, see: a) C. Porcel, P. Lavalle, G. Decher, B. Senger, J. C. Voegel, P. Schaaf, Langmuir 2007, 23, $1898-1904$; b) P. Bieker, M. Schönhoff, Macromolecules 2010, 43, 5052 - 5059; c) L. Xu, D. Pristinski, A. Zhuk, C. Stoddart, J. F. Ankner, S. A.Sukhishvili, Macromolecules 2012, 45, $3892-3901$.

[18] Z. Takats, J. M. Wiseman, B. Gologan, R. G. Cooks, Science 2004, 306, 471- 473. 


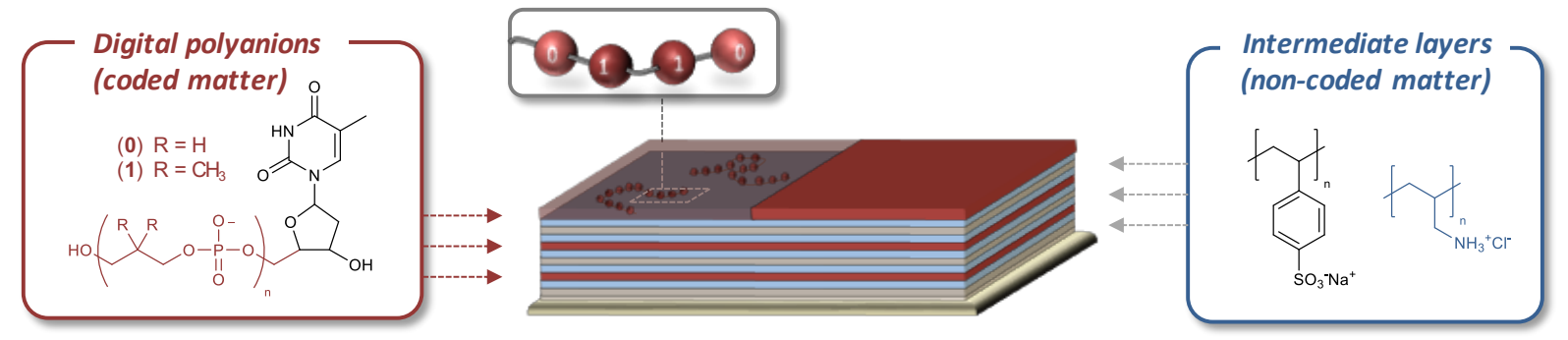

Table of content artwork 